\title{
Jordan-Brans-Dicke Quantum Wormholes and Coleman's Mechanism t
}

\author{
Luis J. Garay ${ }^{\mathbb{H}}$ \\ Instituto de Optica, CSIC \\ Serrano, 121 E-28006 Madrid, Spain \\ Juan García-Bellido: \\ Instituto de Estructura de la Materia , CSIC \\ Serrano, 123 E-28006 Madrid, Spain
}

\begin{abstract}
We consider the quantum gravity and cosmology of a JordanBrans-Dicke theory, predicted by string effective actions. We study its canonical formalism and find that the constraint algebra is that of general relativity, as a consequence of the general covariance of scalar-tensor theories. We also analyze the problem of boundary conditions and propose that they must be imposed in the Jordan frame, in which particles satisfy the strong equivalence principle. Specifically, we discuss both Hartle-Hawking and wormhole boundary conditions in the context of quantum cosmology. We find quantum wormhole solutions for Jordan-Brans-Dicke gravity even in the absence of matter. Wormholes may affect the constants of nature and, in particular, the Brans-Dicke parameter. Following Coleman's mechanism, we find a
\end{abstract}

*Work partially supported by CICYT under contract PB91-0052 and AEN90-0139.

†Supported by MEC-FPI Grant. e-mail: garay@cc.csic.es

${ }^{\ddagger}$ Supported by MEC-FPI Grant. e-mail: bellido@cc.csic.es 
probability distribution which is strongly peaked at zero cosmological constant and infinite Brans-Dicke parameter. That is, we recover general relativity as the effective low energy theory of gravity. 


\section{Introduction}

It is generally believed that the theory of gravity at low energies (general relativity, scalar-tensor, etc.) must be an effective approximation of a more fundamental theory of quantum gravity. Nowadays, the only consistent candidate for such a fundamental theory is string theory [四], based on the assumption that the fundamental objects that describe matter and its interactions are not point-like but one-dimensional. This simple assumption gives a consistent description of gravity at energies above the Planck scale, $M_{\mathrm{Pl}}$, and has very interesting consequences. In particular, the gravitational sector of closed strings contains, apart from the graviton and the antisymmetric tensor field, a dilaton scalar field that couples to gravity and matter. The axion, related to the antisymmetric tensor field, has already been considered in the context of quantum gravity by Giddings and Strominger [2] In this paper we study the scalar component of the gravitational sector in the same context.

The low energy string effective theory can be obtained by integrating out all the string quantum fluctuations with respect to its center of mass [3], giving a local field theory which has the form of a scalar-tensor theory of gravity. We can write the tree level string effective action in four dimensions, keeping only linear terms in the string tension $\alpha^{\prime}$ and in the curvature $R$, as

$$
\tilde{S}=\frac{1}{\alpha^{\prime}} \int d^{4} x \sqrt{-g} e^{-2 \phi}\left(R+4 g^{\alpha \beta} \nabla_{\alpha} \phi \nabla_{\beta} \phi\right)+S_{m},
$$

where $\phi$ is the dilaton field. This action is equivalent to a Jordan-BransDicke (JBD) theory of gravity [1],

$$
\tilde{S}=\frac{1}{16 \pi \mathrm{G}} \int d^{4} x \sqrt{-g}\left(\Phi R-\frac{\omega}{\Phi} g^{\alpha \beta} \nabla_{\alpha} \Phi \nabla_{\beta} \Phi\right)+S_{m},
$$

with a constant Brans-Dicke (BD) parameter, $\omega=-1$. This particular value is due to a fundamental symmetry of strings: target space duality [5]. This is a symmetry of string amplitudes which relates large and small radius of compactification. Therefore, $\omega=-1$ is a model-independent prediction of string theories [6]. One should try to verify phenomenologically this prediction [0], 8]. Jordan-Brans-Dicke theory is based on the idea of Mach that inertia arises from accelerations with respect to the general distribution of 
matter in the universe. Therefore, the inertial masses of elementary particles are not fundamental constants but represent the interaction of particles with a cosmic scalar field whose dynamics depend on the rest of the matter in the universe. Supposing that all matter particles have the same coupling to the scalar field, $m(\phi)=e^{\beta \phi} m$, the action in the so called Einstein frame can be written as

$$
\tilde{S}=\frac{1}{16 \pi \mathrm{G}} \int d^{4} x \sqrt{-\bar{g}}\left(\bar{R}-\frac{1}{2} \bar{g}^{\alpha \beta} \bar{\nabla}_{\alpha} \phi \bar{\nabla}_{\beta} \phi\right)+\int e^{\beta \phi} m d \bar{s} .
$$

Physics must be invariant under conformal redefinitions of the metric since they correspond to an arbitrary choice of measuring units. We are thus free to choose the conformal frame in which we want to describe physics. The most natural choice is the so called physical frame [7], in which observable particles have constant masses, since in this frame particles follow geodesics of the metric and thus satisfy the strong equivalence principle [9]. From the cosmological point of view, the comoving frame, in which a fundamental observer sees the universe as homogeneous and isotropic, is the physical frame since those observers will follow geodesics of the metric. In our case, the conformal redefinition of the metric that allows us to describe the theory of gravitation (1.3) in the physical frame is $\bar{g}_{\alpha \beta}=e^{-2 \beta \phi} g_{\alpha \beta} \equiv \Phi g_{\alpha \beta}$. This is the so called Jordan frame, in which the action takes the form (1.2) where $\Phi$ is a dimensionless scalar field and $\omega$ a constant given by

$$
2 \omega+3=\frac{1}{4 \beta^{2}}
$$

General relativity is recovered in the limit $\omega=\infty,(\beta=0)$. On the other hand, the physical frame for general relativity is the Einstein frame, in which the gravitational action takes the usual Hilbert-Einstein form.

These theories are not ruled out by post-Newtonian experiments [10] nor by primordial nucleosynthesis bounds [11], and in fact have recently recovered great interest since they have been proposed as the arena for extended inflation [12, 13], a new inflationary scenario which could solve some of the traditional problems of previous schemes, such as the graceful exit problem of old inflation [14 and the fine tuning problems of new inflation [15]. Furthermore, as we have mentioned, JBD theory may well be the Planck scale theory of gravity. 
In this paper we will analyze the Jordan-Brans-Dicke quantum cosmology with special emphasis made on the effect of quantum wormholes on the low energy coupling constants. In section 2, we study the canonical formalism of a scalar-tensor theory of gravity. The classical action (1.2) contains second derivatives of the metric tensor, which may be removed with the addition of a suitable surface term [19]. The Hamiltonian constraints can be explicitly written in both the Jordan and Einstein frames. Both expressions are related through a canonical transformation. The constraint algebra is the same in both frames and corresponds to that of general relativity, expressing the fact that JBD theory is generally covariant. Note that a scalar component of gravity does not spoil this covariance.

Boundary conditions must be imposed in the physical frame, where particles satisfy the strong equivalence principle. The boundary conditions in any other frame can be obtained by transforming those in the physical frame through the corresponding conformal redefinition. In JBD quantum cosmology we shall be concerned with boundary conditions both for the universe [20] and for wormholes [21]. The Hartle-Hawking wave function for the universe 22 is given by the Euclidean path integral over all compact fourmetrics in the Jordan frame, over all possible BD field configurations and all matter fields. We find that the saddle point configuration, which gives the dominant contribution to the path integral, will be compact when both the cosmological constant and the BD parameter are positive.

Non-perturbative quantum gravity effects due to non-trivial topologies, e.g. wormholes [23], will change the effective value of the physical parameters of the theory, in particular the Brans-Dicke parameter. Wormholes may be interpreted, semiclassically, as throats joining two otherwise disconnected large regions of spacetime. In this picture one assumes the dilute wormhole approximation, in which wormhole ends are far away from each other and thus can be treated separately. JBD wormhole wave functions can be written as Euclidean path integrals over asymptotically flat spacetimes [21]. One must also sum over all JBD field configurations whose Hamilto-

\footnotetext{
${ }^{1}$ Since the fundamental description of strings gives a JBD theory of gravity below the Planck scale, one should study JBD quantum cosmology, see 16, 17, which may be relevant to the problem of initial conditions for extended inflation. A different approach is string quantum cosmology, see [18], in which the compactification ansatz and the dimension of spacetime are analyzed.
} 
nian vanishes in the asymptotic region and similarly for the matter fields [24].

These boundary conditions have been imposed in the Jordan frame, although in the Einstein frame they take the same form, since the JBD field is constant at infinity. The problem of boundary conditions will be analyzed in detail in section 3 .

Wormholes may play an important role in solving problems associated with the complete evaporation of black holes [25]. Furthermore, although Planck scale wormholes are not directly observable, they will produce effective interactions in the low energy physics [25] that turn the coupling constants of nature into dynamical variables [26, 27]. In particular, JBD wormholes will affect the kinetic term of the JBD field as well as introduce a coupling between this field and particle masses. Both effects will modify the value of the low energy $\mathrm{BD}$ parameter. It is worth noticing that there exist wormhole solutions even in the absence of matter, in contrast with the situation in general relativity. Wormholes in JBD gravity are discussed in section 4 .

Wormholes not only affect the constants of nature but also may provide a probability distribution for them. In general relativity this distribution is strongly peaked at zero cosmological constant. This is the so called Coleman's mechanism [28] for the vanishing of the cosmological constant. In JBD theory an analogous argument leads to the conclusion that not only the cosmological constant vanishes but also the BD parameter is driven to infinity, and consequently there cannot be an effective dynamical scalar field coupled to any form of energy or matter. Thus we recover general relativity as the low energy effective theory of gravity, even though the fundamental high energy description may well be a scalar-tensor theory, as suggested by strings. This result precludes extended inflation, since the predicted values for the cosmological constant and the $\mathrm{BD}$ parameter are the low energy effective values at all times [28]. Section 5 is devoted to Coleman's mechanism in JBD theory.

\section{Canonical formalism}

The Euclidean action for the Jordan-Brans-Dicke theory of gravity, in the Jordan frame, can be obtained through a Wick rotation $(t \rightarrow-i \tau)$ from 
(1.2)

$$
\tilde{I}=\frac{1}{16 \pi \mathrm{G}} \int d^{4} x \sqrt{g}\left\{-\Phi R+\frac{\omega}{\Phi} g^{\alpha \beta} \nabla_{\alpha} \Phi \nabla_{\beta} \Phi\right\}+I_{m}\left[g_{\alpha \beta}, \sigma\right],
$$

where $I_{m}\left[g_{\alpha \beta}, \sigma\right]$ is the Euclidean action of the matter fields $\sigma$. In a $(3+1)$ slicing of spacetime, the metric can be written in the standard from [29, 30]

$$
d s^{2}=\left(N^{2}+N_{i} N^{i}\right) d \tau^{2}+2 N_{i} d x^{i} d \tau+q_{i k} d x^{i} d x^{k},
$$

where $N$ is the lapse function which measures the proper time separation between two neighbouring three-sections, $N^{i}$ are the shift functions which measure the separation between the lines of constant $x^{i}$ and the normal to the three-surface $\Sigma_{\tau}$, and $q_{i k}$ is the metric on this three-surface, which will be chosen to be connected, compact and without boundary. Then, the Euclidean action takes the form

$$
\begin{aligned}
\tilde{I} & =\frac{1}{16 \pi \mathrm{G}} \int d^{3} x d \tau N \sqrt{q}\left\{\Phi\left(K_{i k} K^{i k}-K^{2}\right)-\Phi^{(3)} R\right. \\
& \left.+\frac{\omega}{\Phi}(D \Phi)^{2}+\frac{\omega}{\Phi} q^{i k} \nabla_{i} \Phi \nabla_{k} \Phi-2 \Phi \nabla_{\alpha} f^{\alpha}\right\}+I_{m}\left[g_{\alpha \beta}, \sigma\right]
\end{aligned}
$$

where $q=\operatorname{det} q_{i k}, D \equiv n^{\alpha} \nabla_{\alpha}$ is the derivative in the normal direction to the three-surface, $n^{\alpha}=\left(\frac{1}{N},-\frac{N^{i}}{N}\right)$, and

$$
f^{\alpha}=K n^{\alpha}+D n^{\alpha}
$$

$K$ is the trace of its second fundamental form,

$$
K_{i k}=\frac{1}{2 N}\left(-\dot{q}_{i k}+2 \nabla_{(i} N_{k)}\right)
$$

where an overdot denotes a partial derivative with respect to $\tau$. The expression $\nabla_{\alpha} f^{\alpha}$ contains second time derivatives of the metric. Therefore, the last term in the gravitational Euclidean action can be integrated by parts to give a surface term plus a series of terms which contain only first time derivatives of the three-metric and the JBD field

$$
\begin{aligned}
\tilde{I} & =\frac{1}{16 \pi \mathrm{G}} \int d^{3} x d \tau N \sqrt{q}\left\{\Phi\left(K_{i k} K^{i k}-K^{2}\right)-\Phi^{(3)} R\right. \\
& \left.+\frac{\omega}{\Phi}(D \Phi)^{2}+2 K D \Phi+\omega q^{i k} \nabla_{i} \Phi \nabla_{k} \Phi+2 q^{i k} \nabla_{i} \nabla_{k} \Phi\right\} \\
& +I_{m}\left[q_{i k}, N, N^{i}, \sigma\right]-\frac{1}{8 \pi \mathrm{G}} \int d^{3} x \sqrt{q} \Phi K .
\end{aligned}
$$


In order to be consistent with boundary conditions that fix the three-metric, the JBD and the matter fields at two given three-surfaces [19], we must remove the surface term

$$
I=\tilde{I}+\frac{1}{8 \pi \mathrm{G}} \int d^{3} x \sqrt{q} \Phi K .
$$

Otherwise, we would have to impose the additional requirement that the normal derivatives of the three-metric should also be kept fixed at the boundaries. The Einstein-Jordan-Brans-Dicke and the matter field equations can then be obtained by requiring that the Euclidean action (2.7) be stationary under variations of the fields subjected to the previous boundary conditions.

For the sake of definiteness, we consider a minimally coupled scalar field $\sigma$ as matter content whose action will be

$$
I_{m}\left[q_{i k}, N, N^{i} \sigma\right]=\int d^{3} x d \tau N \sqrt{q}\left\{\frac{1}{2}(D \sigma)^{2}+\frac{1}{2} q^{i k} \nabla_{i} \sigma \nabla_{k} \sigma+V(\sigma)\right\} .
$$

The canonical momenta are given by

$$
\begin{aligned}
& p^{i k}=\frac{\delta I}{\delta \dot{q}_{i k}}=\frac{1}{16 \pi \mathrm{G}} \sqrt{q}\left\{\Phi\left(-K^{i k}+q^{i k} K\right)-q^{i k} D \Phi\right\} \\
& p_{\Phi}=\frac{\delta I}{\delta \dot{\Phi}}=\frac{1}{8 \pi \mathrm{G}} \sqrt{q} \frac{\omega}{\Phi} D \Phi, \\
& p_{\sigma}=\frac{\delta I}{\delta \dot{\sigma}}=\sqrt{q} D \sigma
\end{aligned}
$$

and, therefore, the Hamiltonian of the theory is

$$
H=\int d^{3} x\left(N \mathcal{H}+N^{i} \mathcal{H}_{i}\right)
$$

where the Hamiltonian generators acquire the quite involved expressions

$$
\begin{aligned}
\mathcal{H} & =16 \pi \mathrm{G} \frac{1}{\Phi \sqrt{q}}\left\{p^{i j} p^{k l}\left(q_{i k} q_{j l}-\frac{\omega+1}{2 \omega+3} q_{i j} q_{k l}\right)\right. \\
& \left.-\frac{1}{2 \omega+3} \Phi p_{\Phi} p+\frac{1}{2(2 \omega+3)} \Phi^{2} p_{\Phi}^{2}\right\} \\
& +\frac{1}{16 \pi \mathrm{G}} \sqrt{q}\left\{\Phi^{(3)} R-\omega q^{i k} \nabla_{i} \Phi \nabla_{k} \Phi\right\} \\
& +\frac{1}{2 \sqrt{q}} p_{\sigma}^{2}-\frac{1}{2} \sqrt{q} q^{i k} \nabla_{i} \sigma \nabla_{k} \sigma-\sqrt{q} V(\sigma), \\
\mathcal{H}_{i} & =-2 q_{i k} \nabla_{j} p^{j k}+p_{\Phi} \nabla_{i} \Phi+p_{\sigma} \nabla_{i} \sigma .
\end{aligned}
$$


The constraints of the theory, which together represent the invariance under spatial diffeomorphisms and time reparametrizations, are

$$
\mathcal{H}=0, \quad \mathcal{H}_{i}=0
$$

It can be seen that the Poisson bracket algebra of the constraints is that of general relativity although the direct computation of the constraint algebra is a little messy. However, a simple argument will allow us to obtain this result in a different way. As mentioned in the introduction, due to the equivalence under conformal redefinitions, we can work in the Einstein frame in which the gravitational coupling becomes constant while the matter fields are nontrivially coupled to the JBD scalar. Since this is a non derivative coupling, the canonical structure of the theory will not change. More explicitly, the canonical transformation

$$
\bar{q}_{i k}=\Phi q_{i k}, \quad \phi=-\frac{1}{2 \beta} \log \Phi,
$$

together with the redefinitions of the lapse and shift functions

$$
\bar{N}^{2}=\Phi N^{2}, \quad \bar{N}_{i}=\Phi N_{i},
$$

gives the action in the Einstein frame

$$
\begin{aligned}
\bar{I} & =\frac{1}{16 \pi \mathrm{G}} \int d^{3} x d \tau \bar{N} \sqrt{\bar{q}}\left\{\bar{K}_{i k} \bar{K}^{i k}-\bar{K}^{2}-{ }^{(3)} \bar{R}+\frac{1}{2}(\bar{D} \phi)^{2}+\frac{1}{2} \bar{q}^{i k} \bar{\nabla}_{i} \phi \bar{\nabla}_{k} \phi\right\} \\
& +\bar{I}_{m}\left[\bar{q}_{i k}, \bar{N}, \bar{N}^{i}, \sigma, \phi\right] .
\end{aligned}
$$

Note that the matter action

$\bar{I}_{m}\left[\bar{q}_{i k}, \bar{N}, \bar{N}^{i}, \sigma, \phi\right]=\int d^{3} x d \tau \bar{N} \sqrt{\bar{q}} e^{2 \beta \phi}\left\{\frac{1}{2}(\bar{D} \sigma)^{2}+\frac{1}{2} \bar{q}^{i k} \bar{\nabla}_{i} \sigma \bar{\nabla}_{k} \sigma+e^{2 \beta \phi} V(\sigma)\right\}$

has now an explicit dependence on $\phi$.

The canonical momenta conjugate to the canonical variables $\bar{q}_{i k}, \phi$ and $\sigma$ are

$$
\begin{aligned}
\bar{p}^{i k} & =-\frac{1}{16 \pi \mathrm{G}} \sqrt{\bar{q}}\left(\bar{K}^{i k}-\bar{q}^{i k} \bar{K}\right), \\
\bar{p}_{\phi} & =\frac{1}{16 \pi \mathrm{G}} \sqrt{\bar{q}} \bar{D} \phi, \\
\bar{p}_{\sigma} & =\sqrt{\bar{q}} e^{2 \beta \phi} D \sigma,
\end{aligned}
$$


which can be obtained either directly from the action (2.15) or from (2.9) by means of the canonical transformation (2.13). The Euclidean action in the Einstein frame can then be written in terms of the canonical variables and momenta as

$$
\bar{I}=\int d^{3} x d \tau\left(\bar{p}^{i k} \dot{\bar{q}}_{i k}+\bar{p}_{\phi} \dot{\phi}+\bar{p}_{\sigma} \dot{\sigma}-\bar{N} \overline{\mathcal{H}}-\bar{N}^{i} \overline{\mathcal{H}}_{i}\right)
$$

where the Hamiltonian generators $\overline{\mathcal{H}}$ and $\overline{\mathcal{H}}_{i}$ take the form

$$
\begin{aligned}
\overline{\mathcal{H}} & =16 \pi \mathrm{G} \frac{1}{\sqrt{\bar{q}}}\left(\bar{p}^{i k} \bar{p}_{i k}-\frac{1}{2} \bar{p}^{2}+\frac{1}{2} \bar{p}_{\phi}^{2}\right) \\
& +\frac{1}{16 \pi \mathrm{G}} \sqrt{\bar{q}}\left({ }^{(3)} \bar{R}-\bar{q}^{i k} \bar{\nabla}_{i} \phi \bar{\nabla}_{k} \phi\right) \\
& +e^{-2 \beta \phi} \frac{1}{2 \sqrt{\bar{q}}} \bar{p}_{\sigma}^{2}-\frac{1}{2} e^{2 \beta \phi} \sqrt{\bar{q}} \bar{q}^{i k} \bar{\nabla}_{i} \sigma \bar{\nabla}_{k} \sigma-e^{4 \beta \phi} \sqrt{\bar{q}} V(\sigma), \\
\overline{\mathcal{H}}_{i} & =-2 \bar{q}_{i j} \bar{\nabla}_{k} \bar{p}^{j k}+\bar{p}_{\phi} \bar{\nabla}_{i} \phi+\bar{p}_{\sigma} \bar{\nabla}_{i} \sigma,
\end{aligned}
$$

in terms of which the Hamiltonian of the theory can be written, as before, as

$$
\bar{H}=\int d^{3} x\left(\bar{N} \overline{\mathcal{H}}+\bar{N}^{i} \overline{\mathcal{H}}_{i}\right)
$$

It is straightforward to see that the Poisson bracket algebra of the constraints $\overline{\mathcal{H}}$ and $\overline{\mathcal{H}}_{i}$ is that of general relativity [31]

$$
\begin{aligned}
\left\{\int d^{3} x \bar{N}(x) \overline{\mathcal{H}}(x), \int d^{3} y \bar{M}(y) \overline{\mathcal{H}}(y)\right\} & =-\int d^{3} z\left(\bar{N}(z) \overleftrightarrow{\nabla}^{i} \bar{M}(z)\right) \overline{\mathcal{H}}_{i}(z), \\
\left\{\int d^{3} x \bar{N}^{i}(x) \overline{\mathcal{H}}_{i}(x), \int d^{3} y \bar{M}^{k}(y) \overline{\mathcal{H}}_{k}(y)\right\} & =-\int d^{3} z\left(\overline{\mathcal{L}}_{\vec{N}} \bar{M}^{k}(z)\right) \overline{\mathcal{H}}_{k}(z), \\
\left\{\int d^{3} x \bar{N} \mathcal{\mathcal { H }}(x), \int d^{3} y \bar{M}^{i}(y) \overline{\mathcal{H}}_{i}(y)\right\} & =-\int d^{3} z\left(\overline{\mathcal{L}}_{\vec{M}} \bar{N}(z)\right) \overline{\mathcal{H}}(z),
\end{aligned}
$$

where $\overline{\mathcal{L}}_{\vec{v}}$ is the Lie derivative along the vector $\bar{v}^{i}$. Since the first relation involves the canonical variable $\bar{q}_{i k}$, i.e. $\bar{\nabla}^{i}=\bar{q}^{i k} \bar{\nabla}_{k}$, this algebra is not the Lie algebra of spacetime diffeomorphisms [32]. This was expected since JordanBrans-Dicke theory is generally covariant and we have performed a $(3+1)$ splitting of spacetime, as required by the ADM formalism, which does not preserve the group structure of the four diffeomorphisms. 
The general theory of canonical transformations ensures that, for the transformation (2.13), both Hamiltonians must be equal [33],

$$
H=\int d^{3} x\left(N \mathcal{H}+N^{i} \mathcal{H}_{i}\right)=\int d^{3} x\left(\bar{N} \overline{\mathcal{H}}+\bar{N}^{i} \overline{\mathcal{H}}_{i}\right)=\bar{H}
$$

Since $N$ and $N^{i}$ are just Lagrange multipliers and using (2.14), we find that

$$
\mathcal{H}=e^{-\beta \phi} \overline{\mathcal{H}}, \quad \mathcal{H}_{i}=\overline{\mathcal{H}}_{i},
$$

so that

$$
\int d^{3} x N \mathcal{H}=\int d^{3} x \bar{N} \overline{\mathcal{H}}, \quad \int d^{3} x N^{i} \mathcal{H}_{i}=\int d^{3} x \bar{N}^{i} \overline{\mathcal{H}}_{i}
$$

Finally, the Poisson brackets are also invariant under canonical transformations and, therefore, the algebra of constraints will have the same form (2.21) in both frames. This result was also expected since the change from one frame to the other must not affect the local spacetime structure nor the slicing procedure. It is just a redefinition of the variables on each three-surface without any reference to their embeddings in the spacetime manifold.

\section{Boundary conditions}

In the preceeding section we studied the classical equivalence under canonical transformations of the JBD local dynamical laws, between the Jordan and the Einstein frames. These canonical transformations are in fact conformal redefinitions of the metric. Physics must be invariant under conformal redefinitions of the metric since they correspond to an arbitrary choice of measuring units: physical observables must be constructed as dimensionless variables [4]. In particular, any distance measured in Compton wavelengths of observable particles is invariant under conformal redefinitions [7]. We can therefore arbitrarily choose the conformal frame in which we want to describe physics [34]. However, to describe physical phenomena one not only needs local laws but also boundary conditions that represent global features

of such phenomena. Therefore, two physical theories will be equivalent under conformal redefinitions only if we transform the boundary conditions as well as the local laws. In this section we analyze the boundary conditions for 
the universe and those for wormholes in the context of Jordan-Brans-Dicke quantum cosmology.

The wave function of the universe will be a solution of the WheelerDeWitt equation and the quantum version of the diffeomorphism constraints (2.12) 31

$$
\mathcal{H} \Psi=0, \quad \mathcal{H}_{i} \Psi=0,
$$

in which the classical variables and momenta become operators which act on the wave function by means of the Euclidean correspondence principle. In particular, the momenta can be written as functional derivatives with respect to the canonical variables. The wave function must also satisfy appropriate boundary conditions. We shall be particularly concerned with boundary conditions of the Hartle-Hawking type [22] 6 , since we are interested in Coleman's mechanism [28] in the JBD theory, as will be analyzed in section 5 . The Hartle-Hawking boundary conditions in a JBD theory of gravity can be stated as follows: the wave function of the universe is given by the Euclidean path integral over all compact manifolds and all gravitational and matter fields defined on them which match the arguments of the wave function. As mentioned in the introduction, fundamental observers are in the Jordan frame and therefore the boundary conditions for the universe must be imposed in this physical frame. The boundary conditions in the Einstein frame will be obtained by transforming these in the same way that one transforms the local laws.

For simplicity, let us consider the case of JBD pure gravity plus a cosmological constant. The Euclidean action can then be written as

$$
\tilde{I}=\frac{1}{16 \pi \mathrm{G}} \int d^{4} x \sqrt{g}\left\{-\Phi R+\frac{\omega}{\Phi} g^{\alpha \beta} \nabla_{\alpha} \Phi \nabla_{\beta} \Phi+2 \Lambda\right\}
$$

where $\Lambda$ is the cosmological constant 3 Note that in the Jordan frame the cosmological term is in fact constant and thus satisfies the strong equivalence principle (see also [10]). The saddle point of the Euclidean action, which gives maximum contribution to the path integral, is a solution of the classical

\footnotetext{
${ }^{2}$ Tunneling boundary conditions for the universe in JBD quantum cosmology have recently been discussed by Vilenkin and del Campo 16, 17. In particular, they study the initial conditions for extended inflation.

${ }^{3}$ In the Einstein frame the cosmological term acquires a $\Phi$ dependence given by $\frac{2 \Lambda}{\Phi^{2}}$.
} 
Euclidean equations of motion

$$
\begin{gathered}
R_{\alpha \beta}-\frac{1}{2} g_{\alpha \beta} R=\frac{\Lambda}{\Phi} g_{\alpha \beta}+\frac{\omega}{\Phi^{2}}\left(\nabla_{\alpha} \Phi \nabla_{\beta} \Phi-\frac{1}{2} g_{\alpha \beta}(\nabla \Phi)^{2}\right)+\frac{1}{\Phi}\left(\nabla_{\alpha} \nabla_{\beta} \Phi-g_{\alpha \beta} \nabla^{2} \Phi\right) \\
\nabla^{2} \Phi=\frac{4 \Lambda}{2 \omega+3}
\end{gathered}
$$

The curvature scalar at the saddle point has the form

$$
R=\frac{2 \omega}{2 \omega+3} \frac{4 \Lambda}{\Phi}+\omega\left(\frac{\nabla \Phi}{\Phi}\right)^{2}
$$

It will correspond to a closed universe only when both the cosmological constant $\Lambda$ and the BD parameter $\omega$ are positive. Otherwise, the saddle point configurations are non-compact and therefore are not considered in the path integral. Furthermore, a positive cosmological constant damps the wave function for large four-geometries, while a negative one enhances them [22], and similarly for the BD parameter. We shall therefore consider the case in which $\Lambda>0$ and $\omega>0$.

In the Einstein frame, the saddle point will be compact even for $\omega<0$, since the curvature scalar acquires the form 1

$$
\bar{R}=\frac{4 \Lambda}{\Phi^{2}}+\frac{2 \omega+3}{2}\left(\frac{\bar{\nabla} \Phi}{\Phi}\right)^{2}
$$

In this frame, the vacuum energy $\int d^{3} x \sqrt{\bar{q}(x, t)} \frac{2 \Lambda}{\Phi(x, t)^{2}}$ becomes infinite for negative values of the BD parameter, when the scalar field vanishes. In fact, these spatial surfaces correspond to non-compact three-surfaces in the Jordan frame. On the other hand, for $\omega>0$ the saddle point will be compact in both frames and the vacuum energy finite. This supports the statement that the Jordan frame is the physical frame in which the boundary conditions must be imposed, as stressed above. In fact, imposing Hartle-Hawking boundary conditions in the Einstein frame leads to inconsistencies.

The wormhole wave function will be the solution of both the WheelerDeWitt equation and the quantum diffeomorphism constraints (3.1) subjected to suitable wormhole boundary conditions in the Jordan frame. Since

\footnotetext{
${ }^{4}$ Note that $2 \omega+3$ must be positive in order to ensure that gravity is attractive.
} 
the spacetime is asymptotically Euclidean, the wave function must be exponentially damped for large three-geometries [21]. It must also be regular [21, 35] when the three-geometry degenerates, since the four-geometry is non-singular. The wormhole wave function can also be written as a path integral over all four-geometries which are asymptotically Euclidean and over all possible configurations of the JBD and the matter fields which do not present asymptotic excitations. This means that the gravitational, the JBD and the matter parts of the Hamiltonian must vanish separately in the asymptotic region and, in particular, the JBD field must acquire there a constant homogeneous configuration [24]. Therefore, in the Einstein frame these boundary conditions are expressed in the same form as those in the Jordan frame. Since, in the Einstein frame, the Hamiltonian constraints have a simpler form, the quantization procedure will be carried out in this frame.

\section{Jordan-Brans-Dicke wormholes}

There exist JBD quantum wormhole solutions even in the absence of matter, in contrast with the situation in general relativity. Since the inclusion

of matter may conceal the actual effect of the JBD scalar, we shall study the wormhole solutions of a Jordan-Brans-Dicke theory in vacuum. The matter fields at low energy will feel the existence of JBD wormholes due to the non trivial coupling of the JBD field to their masses, absent in general relativity.

Due to the difficulties attending the full field theoretic expressions that we have to cope with, we shall consider a minisuperspace model [20] in which the metric and the fields are severely restricted so that they depend only on a few functions of time $\tau$. This reduction of the number of degrees of freedom will be carried out by perturbatively expanding the gravitational and the JBD field variables in harmonics on the three-sphere [36]. This corresponds to an approximately homogeneous and isotropic spacetime and thus can be described by a Freedman-Robertson-Walker (FRW) metric plus some perturbations that represent the inhomogeneities and anisotropies. We shall consider that all but the first two modes are in their ground state so that we can write the three-metric, the lapse and shift functions and the 
JBD field as

$$
\begin{aligned}
\bar{q}_{i k}\left(\tau, x^{i}\right) & =\frac{2 \mathrm{G}}{3 \pi} e^{2 \alpha(\tau)}\left(1+\sqrt{\frac{2}{3}} a(\tau) Q^{(2)}\left(x^{i}\right)\right) \Omega_{i k}\left(x^{i}\right), \\
\bar{N}\left(\tau, x^{i}\right) & =\sqrt{\frac{2 \mathrm{G}}{3 \pi}} N_{0}(\tau)\left(1+\frac{1}{\sqrt{6}} g(\tau) Q^{(2)}\left(x^{i}\right)\right), \\
\bar{N}_{i}\left(\tau, x^{i}\right) & =\frac{2 \mathrm{G}}{3 \pi} e^{\alpha(\tau)}\left(\frac{1}{\sqrt{6}} k(\tau) P_{i}^{(2)}\left(x^{i}\right)\right), \\
\phi\left(\tau, x^{i}\right) & =\frac{3 \pi}{2 \mathrm{G}}\left(\frac{1}{\pi} \varphi(\tau)+\sqrt{2} f(\tau) Q^{(2)}\left(x^{i}\right)\right),
\end{aligned}
$$

where $\Omega_{i k}$ and $x^{i}$ are the metric and the coordinates on the unit threesphere, $e^{\alpha}$ is the usual scale factor in a FRW spacetime, $N_{0}$ and $\varphi$ are the homogeneous modes of the lapse function and the JBD field respectively, and $a, g, k$ and $f$ are the coefficients of the expansion. $Q^{(2)}$ are the standard scalar harmonics on the three-sphere and $P_{i}^{(2)}=\bar{\nabla}_{i} Q^{(2)}$ are the transverse vector harmonics that correspond to the first inhomogeneous mode $n=2$. In the expansion of the three-metric $\bar{q}_{i k}$, all the other terms, which correspond to the transverse traceless vector and tensor harmonics, have not been considered since they will not appear in the action [36].

The Euclidean action, up to second order in the perturbations, will then be the sum of the zeroth order action corresponding to the homogeneous mode (i.e. to a r FRW spacetime plus the homogeneous JBD field), and the action associated to the first inhomogeneous mode, quadratic in the perturbations [36]. One can then obtain the classical Hamiltonian of the theory

$$
\bar{H}=N_{0}\left(H_{\mid 0}+H_{\mid 2}^{(2)}+g H_{\mid 1}^{(2)}\right)+k H_{-1}^{(2)},
$$

where the subindex gives the order in perturbation theory. Since $N_{0}, k$ and $g$ are not dynamical, they can be freely varied to yield, by replacing the momenta by the derivatives with respect to the canonical variables, the quantum Hamiltonian constraints

$$
H_{-1}^{(2)} \Psi=0, \quad H_{\mid 1}^{(2)} \Psi=0, \quad\left(H_{\mid 0}+H_{\mid 2}^{(2)}\right) \Psi=0 .
$$

The explicit expressions of these operators can be found in ref. [36]. The first one is the three-diffeomorphism constraint, which ensures that the wave 
function is invariant under spatial changes of coordinates while the last two correspond to the Wheeler-DeWitt equation. Since the diffeomorphism constraint is linear in the gravitational momentum $p_{a}$, we can substitute for $p_{a}$ and then solve the resultant Wheeler-DeWitt equations in the gauge $a=0$,

$$
\begin{gathered}
\partial_{\varphi}\left(\partial_{f}-3 f \partial_{\alpha}\right) \Psi=0 \\
\left(\partial_{\alpha}^{2}-\left(1-9 f^{2}\right) \partial_{\varphi}^{2}-\partial_{f}^{2}-\left(1-3 f^{2}\right) e^{4 \alpha}\right) \Psi=0 .
\end{gathered}
$$

One can use the solution to these equations as an initial value for the diffeomorphism constraint equation and, thus, find the wave function for any value of $a$ [36]. Although these equations are formally equivalent to those of a minimally coupled massless scalar field in general relativity, $\varphi$ is actually the homogeneous scalar component of the gravitational field, rather than a matter field.

For the wave function $\Psi$ to describe a wormhole, the JBD field must be homogeneous at infinity, with an asymptotically zero energy-momentum tensor [24]. This means that the perturbations must vanish in the asymptotic region. Then equations (4.4, 4.5) reduce to those of the unperturbed model,

$$
\left(\partial_{\alpha}^{2}-\partial_{\varphi}^{2}-e^{4 \alpha}\right) \Psi=0
$$

whose wormhole solutions are well known [21, 24]. In fact one can construct a complete set of wave functions that generates the whole Hilbert space of quantum wormholes [35]. Far away from the asymptotically euclidean region, the contribution of the perturbations become important and, therefore, the wormhole wave function will have an explicit non-trivial dependence on them that cannot be gauged away, see however [37. We have not found explicit analytic expressions for these solutions. However, a complete set of solutions must exist, due to the existence of such a set in the unperturbed model.

Particles may go down the wormhole from one asymptotically Euclidean region to another. This can be interpreted from one of these regions as an effective interaction which modifies the low energy coupling constants. More precisely, the Green's function between these two regions can be factorized by inserting a complete set of wormhole solutions of the Wheeler-DeWitt equation [25]. The two-point wormhole vertex can then be written as the matrix element between the ordinary flat space vacuum $|0\rangle$ and the wormhole 
state $\Psi_{\text {wh }}$

$$
\left\langle 0\left|\phi\left(x_{1}\right) \phi\left(x_{2}\right)\right| \Psi_{\mathrm{wh}}\right\rangle=\int \mathcal{D} \bar{q}_{i k}^{\prime} \mathcal{D} \phi^{\prime} \Psi_{\mathrm{wh}}\left(\bar{q}_{i k}^{\prime}, \phi^{\prime}\right) \int \mathcal{D} \bar{g}_{\alpha \beta} \mathcal{D} \phi \phi\left(x_{1}\right) \phi\left(x_{2}\right) e^{-\bar{I}},
$$

where the latter path integral is over all asymptotically Euclidean fourmetrics $\bar{g}_{\alpha \beta}$ and all JBD fields $\phi$ that have vanishing energy-momentum tensor at infinity, which match the values $\bar{q}_{i k}^{\prime}$ and $\phi^{\prime}$ on a given three-surface. This path integral can be evaluated in the saddle point approximation. From the classical equations of the homogeneous and inhomogeneous parts of the JBD field

$$
\dot{\varphi}+2 \varphi \dot{\alpha}=0, \quad \dot{f}+3 f \dot{\alpha}=0,
$$

respectively, it can be seen that the stationary JBD configuration falls off like 39]

$$
\phi_{\text {hom }}(x) \sim \frac{1}{\left|x-x_{0}\right|^{2}}, \quad \phi_{\text {inh }}(x) \sim \frac{Q^{(2)}\left(x^{i}\right)}{\left|x-x_{0}\right|^{3}},
$$

for large distance $\left|x-x_{0}\right|$ from the center $x_{0}$ of the wormhole. Therefore, the two-point wormhole vertex associated with the inhomogeneous mode can be found to be

$$
\left\langle 0\left|\phi\left(x_{1}\right) \phi\left(x_{2}\right)\right| \Psi_{\mathrm{wh}}\right\rangle=\text { factor } \times \int d^{4} x_{0} \frac{Q^{(2)}\left(\left(x_{1}-x_{2}\right)^{i}\right)}{\left|x_{1}-x_{0}\right|^{3}\left|x_{2}-x_{0}\right|^{3}},
$$

where an integration over all possible orientations of the wormhole has been performed and the prefactor depends on the wormhole quantum state. This is precisely the kind of vertex that one would obtain from an interaction in flat spacetime of the form $\int d^{4} x_{0}\left(\partial \phi\left(x_{0}\right)\right)^{2}$. Furthermore, in the presence of low energy matter, wormholes produce an effective interaction which modifies the masses of elementary particles with an effective coupling to the scalar field 0 . Both effects can be reinterpreted, through a conformal redefinition of the metric, as giving an effective $\omega$ parameter.

\footnotetext{
${ }^{5}$ In an analogous way, wormholes will induce an effective potential for the scalar field. However, observational bounds put very strong constraints on its mass. From now on, we will ignore this contribution.
} 


\section{The effective Brans-Dicke parameter}

As explained in the introduction, string theory predicts an effective JBD theory of gravity with a particular value, $\omega=-1$, of the BD parameter. One should verify phenomenologically this prediction [8]. Soon after the Planck era, a phase transition due to a symmetry breaking could be responsible for a short period of extended inflation [12]. In a homogeneous and isotropic spacetime $d s^{2}=-d t^{2}+e^{2 \alpha(t)} d \Omega_{3}^{2}$, the cosmological solutions to the Lorentzian Jordan-Brans-Dicke equations of motion (which can be obtained from (3.3)), follow the power law evolution 40

$$
e^{\alpha(t)} \sim t^{\omega+\frac{1}{2}}, \quad \Phi(t) \sim t^{2}
$$

A value of $\omega \gg 1$ is necessary for solving the horizon and flatness problems [12] 6. String theory prediction, $\omega=-1$, would give a contraction of the universe, see eq. (5.1), instead of an expansion. Furthermore, this value of $\omega$ is also in conflict with primordial nucleosynthesis and post-Newtonian bounds, $\omega>500$ [11, 10]. We therefore seem to be very far from the string fundamental description of gravity.

Nevertheless, one expects that non-perturbative quantum gravity wormhole effects modify the effective value of the Brans-Dicke parameter, as explained in section 4. We will now follow Coleman's arguments for the vanishing of the cosmological constant 28, 41, 42] in the context of a JBD theory of gravity. We will obtain that the most probable value of the effective BD parameter is $\omega=\infty$, that is, we recover general relativity as the low energy effective theory of gravity.

We cannot observe wormholes, since they do not carry any gauge charge, energy or momentum [25]. They are topological quantum fluctuations of Euclidean spacetime which connect separate universes. The effect of wormholes can be seen as an insertion in the field theory path integral of an apparently non-local vertex operator which can then be rewritten as an effective local interaction that modifies the low energy coupling constants [41]. These will depend on wormhole configurations labelled by arbitrary $\alpha$ parameters. The

\footnotetext{
${ }^{6}$ However, in order not to disturb the observed isotropy of the cosmic background radiation, we must also require $\omega \lesssim 25[12$.
} 
Euclidean path integral for the whole universe gives a probability distribution for these $\alpha$ parameters and, as a consequence, for the coupling constants of nature

$$
\mathcal{Z}=\int d \alpha e^{-\frac{1}{2} \alpha^{2}} \mathcal{Z}(\alpha)
$$

where the weight $\exp \left(-\alpha^{2} / 2\right)$ is determined by the Hartle-Hawking boundary conditions for the universe [28], which were discussed in section 3. If this probability distribution is strongly peaked at particular values of these coupling constants, they are a prediction of the theory in the sense that these will be the value we will observe, with very high probability. Coleman assumes that in the path integral one must sum over all closed manifolds subjected to Hartle-Hawking boundary conditions. He further assumes that wormholes do not interfere (dilute wormhole approximation). The partition function $\mathcal{Z}(\alpha)$ is given by the Euclidean path integral over all compact manifolds, containing large smooth compact regions of spacetime disconnected from each other, except for the existence of wormholes, which can be written as the exponential of the path integral over compact connected manifolds,

$$
\mathcal{Z}(\alpha)=\sum_{\mathrm{CM}} \int \mathcal{D} g \mathcal{D} \Phi e^{-I(\alpha)}=\exp \sum_{\mathrm{CCM}} \int \mathcal{D} g \mathcal{D} \Phi e^{-I(\alpha)} .
$$

He then assumes that the most important contribution to the path integral comes from manifolds with spherical topology. Under those assumptions one can then evaluate the path integral in the saddle point approximation.

We will evaluate this path integral in the JBD theory, see eq. (3.2). As discussed in section 3, Hartle-Hawking boundary conditions must be imposed in the Jordan frame. We saw that the Euclidean saddle point corresponds to a compact spacetime only if both the cosmological constant and the BD parameter are positive. The Euclidean action at the saddle point can be computed numerically, from the eqs. (3.3), with the use of Mathematica [43]. The resulting effective saddle point action can be written as

$$
I(\alpha)=-\frac{3 \pi}{\mathrm{G}_{\alpha} \Lambda_{\alpha}} f\left(\omega_{\alpha}\right),
$$

where $f\left(\omega_{\alpha}\right)$ is the function shown in fig. 1 , whose maximum value is obtained for $\omega_{\alpha}=\infty$, where $f(\infty)=1$, for which we recover the general relativity result. 
The probability distribution for the $\alpha$ parameters and therefore for the coupling constants

$$
\mathcal{Z}(\alpha)=\exp \left(\exp \frac{3 \pi}{\mathrm{G}_{\alpha} \Lambda_{\alpha}} f\left(\omega_{\alpha}\right)\right) .
$$

is strongly peaked at $\mathrm{G}_{\alpha} \Lambda_{\alpha}=0$. Since this probability distribution is not normalizable, a suitable choice of the cutoff is needed in order to determine its maximum. In the context of general relativity, different cutoffs have been considered [28, 42, 44] that give different results. However, G $\Lambda$ seems to be the most natural choice for the cutoff since it is the adimensional vacuum energy, i.e. the cosmological constant in Planck units, which is observed to be less than $10^{-120}$. With this choice for the cutoff, the probability distribution (5.5) acquires its maximum at $\omega_{\alpha}=\infty$. Therefore, general relativity is a prediction of this scenario based on non-perturbative quantum gravity effects. This means that there can be no effective dynamical scalar field coupled to energy or matter. In particular, even in the case that different kinds of matter had different couplings to the scalar field [45, 7, 8], the weak equivalence principle will be effectively recovered due to wormholes. Note that this result can be generalized to any scalar-tensor theory with an arbitrary coupling $\omega(\Phi)$.

It is important to understand the physical meaning of this prediction. The usual

interpretation [28] is that Coleman's mechanism only ensures that the bottom line cosmological constant is zero. This does not exclude an inflationary universe with a non-zero false vacuum energy due a certain phase transition. It just says that, whatever the effective potential for inflation is, the true minimum is at zero cosmological constant. However, it does preclude extended inflation since wormholes drive the BD parameter to infinity at all times and thus "freeze out" the evolution of the scalar field.

\section{Acknowledgments}

We would like to thank Pedro González-Díaz, Guillermo Mena Marugán and Mariano Quirós for a careful reading of the manuscript and valuable

discussions. We also thank Andrei Linde for clarifying our conclusions on extended inflation. 


\section{References}

[1] See for instance, M. B. Green, J. H. Schwarz and E. Witten, Superstring Theory Cambridge Univ. Press (1987).

[2] S. B. Giddings and A. Strominger, Nucl. Phys. B 306 (1988) 890.

[3] E. S. Fradkin and A. A. Tseytlin, Nucl. Phys. B 261 (1985) 1; Phys. Lett. B 158 (1985) 316.

[4] P. Jordan, Nature (London) 164 (1949) 637; Z. Phys. 157 (1959) 112; C. Brans and R. H. Dicke, Phys. Rev. 124 (1961) 925; R. H. Dicke, Phys. Rev. 125 (1962) 2163; C. Brans, Phys. Rev. 125 (1962) 2194.

[5] K. Kikkawa and M. Yamasaki, Phys. Lett. B 149 (1984) 357; N. Sakai and I. Senda, Prog. Theor. Phys. 75 (1986) 692; V. Nair, A. Shapere, A. Strominger and F. Wilczek, Nucl. Phys. B 287 (1987) 402.

[6] J. A. Casas, J. García-Bellido and M. Quirós, Nucl. Phys. B 361 (1991) 713.

[7] J. A. Casas, J. García-Bellido and M. Quirós, Class. Quant. Grav. 9 (1992) 1371.

[8] J. García-Bellido, Gravitation and Cosmology from String Effective Theories, Ph. D. Thesis (1992).

[9] See for instance, S. Weinberg, Gravitation and Cosmology (Wiley, New York, 1972).

[10] For a general review see C. M. Will, Theory and Experiment in Gravitational Physics, Cambridge University Press (1981); Phys. Rep. 113 (1984) 345 .

[11] J. A. Casas, J.García-Bellido and M. Quirós, Mod. Phys. Lett. A 7 (1992) 447; Phys. Lett. B 278 (1992) 94.

[12] D. La and P. J. Steinhardt, Phys. Rev. Lett. 62 (1989) 376; Phys. Lett. B 220 (1989) 375; E. J. Weinberg, Phys. Rev. D 40 (1989) 3950; D. La, P. J. Steinhardt and E. W. Bertschinger, Phys. Lett. B 231 (1989) 231; J. García-Bellido and M. Quirós, Phys. Lett. B 243 (1990) 45. 
[13] J. García-Bellido and M. Quirós, Nucl. Phys. B 368 (1992) 463.

[14] A. H. Guth, Phys. Rev. D 23 (1981) 347; A. H. Guth and E. J. Weinberg, Nucl. Phys. B 212 (1983) 321.

[15] A. D. Linde, Phys. Lett. B 108 (1982) 389; A. Albrecht and P. J. Steinhardt, Phys. Rev. Lett. 48 (1982) 1220.

[16] S. del Campo and A. Vilenkin, Phys. Rev. D 40 (1989) 688; S. del Campo, Phys. Lett. B 259 (1991) 34.

[17] A. K. Sanyal and B. Modak, Int. J. Mod. Phys. A 7 (1992) 4039.

[18] K. Enqvist, S. Mohanty and D. V. Nanopoulos, Phys. Lett. B 192 (1987) 327; Int. J. Mod. Phys. A 4 (1989) 873; J. Wang and S. Mohanty Int. J. Mod. Phys. A 6 (1991) 2181; M. D. Pollock, Nucl. Phys. B 315 (1989) 528; Nucl. Phys. B 324 (1989) 187; Int. J. Mod. Phys. A 7 (1992) 4149.

[19] S. W. Hawking, in General Relativity: An Einstein Centenary Survey, Eds. S. W. Hawking and W. Israel, Cambridge U.P., Cambridge (1979).

[20] J. J. Halliwell, in Proceedings of the Seventh Jerusalem Winter School for Theoretical Physics: Quantum Cosmology and Baby Universes, Ed. T. Piran (1990).

[21] S. W. Hawking and D. N. Page, Phys. Rev. D 42 (1990) 2655.

[22] J. B. Hartle and S. W. Hawking, Phys. Rev. D 28 (1983) 2960.

[23] S. W. Hawking, Mod. Phys. Lett. A 5 (1990) 145; Mod. Phys. Lett. A 5 (1990) 453.

[24] L. J. Garay, Phys. Rev. D 44 (1991) 1059.

[25] S. W. Hawking, Phys. Rev. D 37 (1988) 904.

[26] S. W. Hawking and R. Laflamme, Phys. Lett. B 209 (1988) 39.

[27] S. Coleman, Nucl. Phys. B 307 (1988) 864.

[28] S. Coleman, Nucl. Phys. B 310 (1988) 643. 
[29] R. Arnowitt, S. Deser and C. W. Misner, in Gravitation: An Introduction to Current Research, Ed. L. Witten, Wiley, New York (1962).

[30] C. W. Misner, K. S. Thorne and J. A. Wheeler, Gravitation Freeman, San Francisco (1973).

[31] K. Kuchař, in Quantum Gravity 2: A Second Oxford Symposium, Eds. C. J. Isham, R. Penrose and D. W. Sciama, Clarendon P., Oxford (1981).

[32] C. J. Isham and K. Kuchař, Ann. Phys. 164 (1985) 288; Ann. Phys. 164 (1985) 316;

[33] H. Goldstein, Classical Mechanics, Addison Wesley, Reading MA (1959).

[34] S. W. Hawking, Comm. Math. Phys. 25 (1972) 167.

[35] L. J. Garay, Wormholes in Quantum Cosmology, Ph. D. Thesis (1992).

[36] J. J. Halliwell and S. W. Hawking, Phys. Rev. D 31 (1985) 1777.

[37] A. Lyons, "All-order wormhole vertex operators from the WheelerDeWitt equation" preprint ALBERTA-THY-3-92.

[38] L. M. Campbell and L. J. Garay, Phys. Lett. B 254 (1991) 49.

[39] H. F. Dowker, Nucl. Phys. B 331 (1990) 194.

[40] C. Mathizhagan and V. B. Johri, Class. Quant. Grav. 1 (1984) L29.

[41] I. Klebanov, L. Susskind and T. Banks, Nucl. Phys. B 317 (1989) 665.

[42] S. W. Hawking, Nucl. Phys. B 335 (1990) 155.

[43] S. Wolfram, Mathematica, 2nd Ed., Addison-Wesley (1991).

[44] J. Preskill, Nucl. Phys. B 323 (1989) 141; B. Grinstein and M. B. Wise, Phys. Lett. B 212 (1988) 407; B. Grinstein, Nucl. Phys. B 321 (1989) 439.

[45] T. Damour, G.W. Gibbons and C. Gundlach, Phys. Rev. Lett. 64 (1990) 123. 
Fig.1 Plot of the function $f(\omega)$ which determines the saddle point action (5.4). The $\omega<0$ region has been excluded since it corresponds to noncompact saddle points. This function acquires its maximum at $\omega=\infty$, where $f(\infty)=1$, which corresponds to the general relativity result. 